\title{
Numerical analysis of surface cracks repaired with single and double patches of composites
}

\author{
Inés Iváñez' and Matías Braun ${ }^{2,3}$
}

\begin{abstract}
In this work, the performances of circular single- and double-sided composite patch repairs are compared by computing the maximum stress intensity factor of a repaired surface crack. The three-dimensional finite-element method is used to calculate the stress intensity factor along the crack. The effects of the crack depth, composite patch thickness and patch material on the stress intensity factor variation are highlighted. The obtained results show that the selection of single- or double-sided patches depends on both the crack depth and patch thickness.
\end{abstract}

\section{Keywords}

Surface crack, stress intensity factor, finite-element method, composite

\section{Introduction}

Bonded composite repair has many advantages over a mechanically fastened, which include lower density, improved fatigue behaviour, reduced costs, and excellent formability. ${ }^{1,2}$ It also provides more uniform and efficient load transfer into the repair patch and less stress concentration. ${ }^{3} \mathrm{Up}$ until today, however, the certification rules applicable for primary bonded structures prevent the use of boltless bonded joints; ${ }^{4}$ therefore, the need to gain knowledge about their behaviour under different conditions and crack types is apparent, and has been a topic of several researches during the last two decades.

The structural stability of bonded composite repairs depends on their mechanical performance during their service life, and most reports dealing with design of bonded patches emphasise the need to have an accurate tool for evaluating repairs against all criteria, measuring crack resistance curves, ${ }^{5}$ and predicting stress intensity factors (SIF) and failure strength. ${ }^{6}$ There are several methods to perform this analysis, which can be divided into two main groups: analytical and numerical. In general, the analytical method uses elasticity solutions and provides accurate solutions with a small amount of inputs; ${ }^{3}$ although, to predict the failure load of a patch-repaired structure, a 3D configuration is required as the stress results can be used to identify critical regions. ${ }^{7}$ As a result, due to the increase of computational power, the finite element method has contributed considerably to the knowledge of the mechanical behaviour of defects under patch repairs and has been used by many authors, ${ }^{8-10}$ finding a detailed review of its application to adhesively bonded repair in Xiaocong. ${ }^{11}$

Considering the fact that the geometry and materials of a repair patch are usually determined at an initial design stage and cannot be easily changed, optimisation of repairs should be feasible and reasonable. Some design parameters that have been identified to play important roles in bonded composite patches include: patch size, shape, material selection, and adhesive properties. $^{12}$

A way to design a patch repair can be performing a study of its optimal shape, in order to obtain the

\footnotetext{
'Department of Continuum Mechanics and Structural Analysis, University Carlos III of Madrid, Spain

${ }^{2}$ Departamento de Construcciones, Facultad de Ingeniería, Universidad Nacional de La Plata, Argentina

${ }^{3}$ Consejo Nacional de Investigaciones Científicas y Técnicas (CONICET), CCT La Plata, Argentina
}

\section{Corresponding author:}

Inés Ivañez, University Carlos III of Madrid, Avda. de la Universidad 30, Leganes, Madrid 289II, Spain.

Email: idel@ing.uc3m.es 
maximum safety-cost ratio. ${ }^{13,14}$ In this regard, Ouinas et al. ${ }^{15}$ have analysed the performance of octagonal, circular, and elliptical shapes of patches, using the finite-element method. They concluded that the patch shape has a significant effect on the value of the SIF at the crack tip. Ramji et al. ${ }^{9}$ have compared the repair performance of patches with circle, rectangle, square, ellipse, and octagon shapes by using the finite-element method, and they found that the octagonal and rectangular patch shapes were better for mixed-mode problems. In spite of the wide range of shapes studied, only double-sided patch repairs were analysed.

The use of single- and double-sided patch repairs has been widely covered in literature, and many authors have studied the influence of both patch configurations on the performance of externally bonded patch repairs. Ramji and Srilakshmi ${ }^{16}$ have carried out a threedimensional finite element analysis to study and compare the performance of single- and double-sided patch on center-cracked aluminum panel. They have shown that the mechanics of both type of repairs is completely different, and concluded that double-sided patch is more efficient. They also highlighted that in case of the single-sided repairs, $\mathrm{K}_{\mathrm{I}}$ at the unpatched surface exceeded the value obtained in unrepaired panels due to out-of-plane bending. Ouinas et al. ${ }^{17}$ have conducted a numerical analysis for studying the repairing cracks from a semicircular lateral notch, determining the SIF for different inclination angles. They observed that the double-sided repair allowed the elimination of the mentioned bending effect, but led to a gain in thickness that was not neglegible in both mode I and mixed mode cases. Albedah et al. ${ }^{18}$ have used finite element analysis to estimate SIF for single- and double-sided repairs having a circular patch shape. They concluded that the mass gain given by the double-sided repair can be very significant, depending on both the patch shape and the adhesive properties, and emphasised the difficulty to follow the crack propagation of double-sided repairs. Regarding the adhesive, Bachir Bouiadjra et al. ${ }^{19}$ analysed the effect of a disband occurring for cracks repaired with boron/epoxy patch, and they concluded that the SIF is increased by the presence of the adhesive disband.

Thin repaired plates have been modelled in the aforementioned works to represent the skin of an aircraft and a reduction in SIF while using double-sided patches has been observed; nevertheless, not all reparable structures are accessible from both sides, and therefore double-sided patches are not suitable for all applications. The increasing use of composite patching for naval applications has led some studies to consider the use of patches in thicker plates, ${ }^{20,21}$ and the behaviour of thicker repaired components can be different from those observed in thinner plates.
These previous works focus on analysing the performance of structures with repaired straight-fronts cracks, although fatigue failures in structures usually occur from the initiation and propagation of cracks from notches or defects in the material that is either embedded or at a corner, which propagate with elliptic or near-elliptic cracks fronts. ${ }^{22,23}$ There is a lack of knowledge in the analysis of the mechanical response of thick metallic panels with through-the-thickness cracks, which initiate near regions of stress concentrations and may cause premature failure of aircraft landing gears, spars, stiffeners, and other components. ${ }^{24}$ In this context, realistic assessment of failure conditions requires an accurate determination of SIF and a detailed study of the patch performance.

In the present study, a linear 3D finite-element analysis is performed to investigate the influence of bonded patch repairs on the performance of a thick metallic cracked panel. The study is conducted for aluminium panels and the composite patch is of UD configuration. Different geometries, materials, and crack depths are considered. Particular attention is given to the performance of double-sided compared to single-sided patches.

\section{Geometrical and material properties}

The basic geometry of the cracked structure under study is shown in Figure 1, in which a rectangular elastic aluminium plate with a central surface crack is subjected to a remote uniaxial tensile load in the vertical direction (stress amplitude $=70 \mathrm{MPa}$ ). The plate is made of aluminum alloy T3 of dimensions $254 \times 254 \times 12 \mathrm{~mm}^{3}$, and it contains a semi-elliptical crack of length $2 a$ and depth $c$, as detailed in Figure 1(a). The thickness of the plate is taken from the experimental work presented by Jones and Chiu. ${ }^{25}$

The crack is repaired with a bonded unidirectional boron/epoxy composite patch, in which the fibre

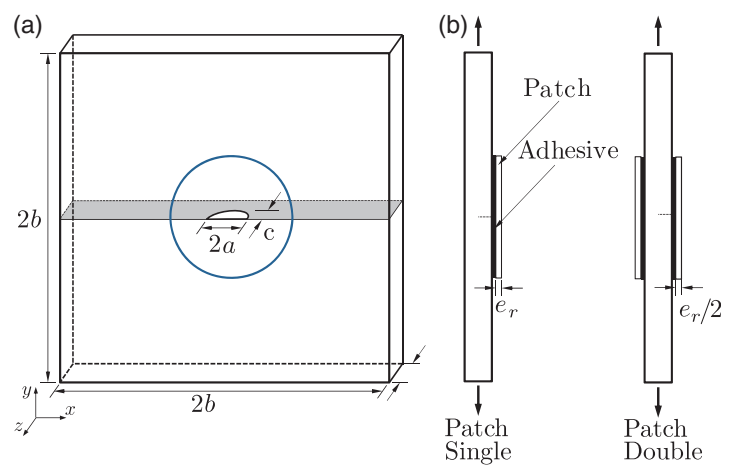

Figure I. Geometry of the model: (a) perspective view and (b) side view. All dimensions are in $\mathrm{mm}$. 
direction is parallel to the direction of the load. Figure 1(b) shows that the thickness of double-sided patch is half of that of the single-sided, following the same procedure presented by Albedah et al. ${ }^{18}$

The composite patch is bonded to the cracked plate with an FM 73 epoxy adhesive, having a thickness of $0.15 \mathrm{~mm} .{ }^{6,18}$ General material properties of the cracked plate, the adhesive, and the composite patch are given in Table 1 . The considered geometry and material properties are taken from the work presented by Albedah et al. ${ }^{18}$

\section{Finite-element model}

A three-dimensional model is implemented using the commercial finite-element code Abaqus/Standard. ${ }^{26}$ The finite-element model consists of three parts: cracked plate, adhesive, and composite patch. The symmetry of the problem permitted to represent only a quarter of the repaired structure, therefore the size of the analysis domain and the analysis run time were reduced (Figure 2).

The contact between the different parts is assumed to be perfect ${ }^{9,10}$ and modelled by means of tie constraints, which are available in the Abaqus/Standard library. ${ }^{26}$ The aluminium plate was pulled in tension, $\sigma=70 \mathrm{MPa}$.

To capture the high stress gradient existing along the crack tip, the mesh has been refined around this area, where the element size is reduced to a dimension of $0.015 \mathrm{~mm}$. Before performing further simulations, the sensitivity of the mesh was evaluated by carrying out successive space discretisations and analysing the mode I SIF of the repaired structure. The SIF is calculated using the J-integral, which is based on the virtual crack extension method available in the commercial FE

Table I. Mechanical properties of aluminium, composite and adhesive.

\begin{tabular}{llll}
\hline & \multicolumn{2}{l}{ Material } \\
\cline { 2 - 4 } Properties & Aluminium & Boron/epoxy & Adhesive \\
\hline$E_{11}-(\mathrm{GPa})$ & 72 & 210 & 2.55 \\
$\mathrm{E}_{22}-(\mathrm{GPa})$ & - & 19.6 & - \\
$\mathrm{E}_{33}-(\mathrm{GPa})$ & - & 19.6 & - \\
$v_{12}$ & 0.33 & 0.3 & 0.32 \\
$v_{13}$ & - & 0.28 & - \\
$v_{23}$ & - & 0.28 & - \\
$\mathrm{G}_{12}-(\mathrm{GPa})$ & - & 7.2 & - \\
$\mathrm{G}_{13}-(\mathrm{GPa})$ & - & 5.46 & - \\
$\mathrm{G}_{23}-(\mathrm{GPa})$ & - & 5.46 & - \\
\hline
\end{tabular}

package Abaqus. For each point of the crack, ten integration contours are computed. Each contour is defined in the normal direction of the semi-elliptical crack front.

The quarter-elliptical surface crack is meshed with 45 elements along its perimeter, hence 45 values of SIF are obtained along the crack tip. For each studied configuration, the result presented corresponds to the maximum value of SIF obtained in each simulation. The adhesive is meshed with one solid element through its thickness, and the patch is meshed with four solid elements through the thickness. The aluminum plate and the adhesive are meshed with 336440 and 9858 elements, respectively, while the patch is discretised with 9858 elements for each milimeter of thickness. All parts are meshed with C3D8R elements (continuum three-dimensional reduced integration eight node elements).

\section{Results and discussion}

The objective of this work is to compare the performances of circular single- and double-sided patch repairs by computing the maximum SIF in mode I at a repaired

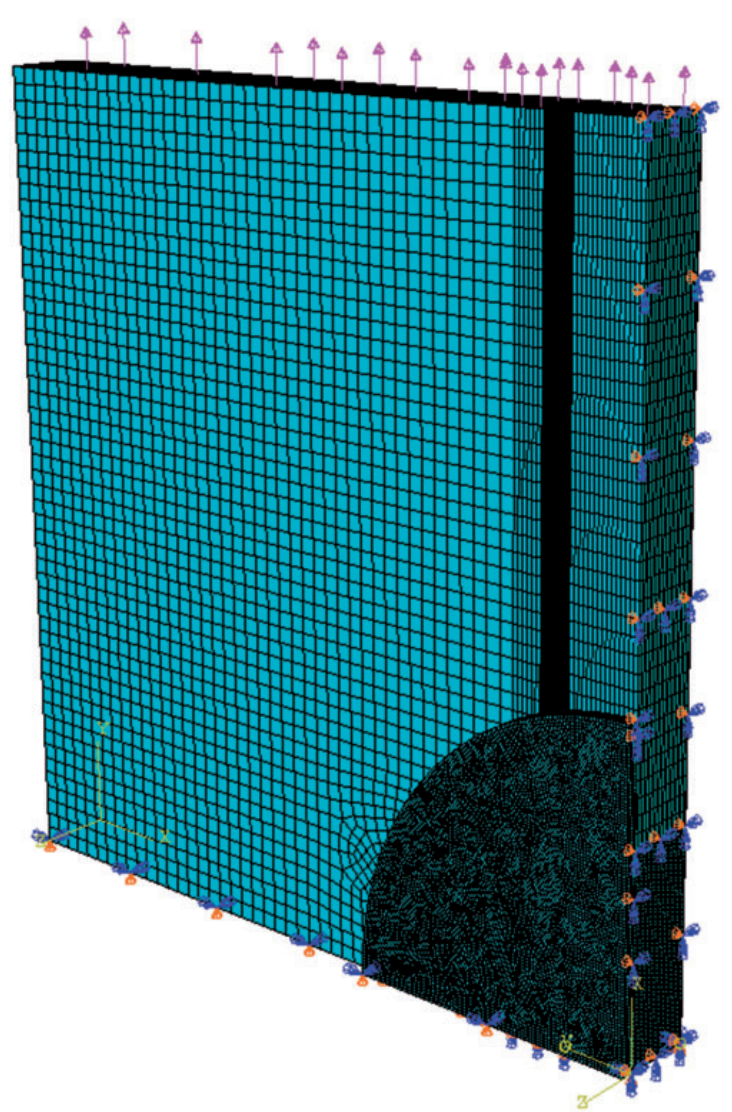

Figure 2. Typical mesh of the finite-element model of the repaired structure. 
surface crack. The results obtained are presented and analysed in the following subsections.

\section{Mesh validation: comparison between analytical and numerical SIF}

In this subsection, the three-dimensional numerical model of the unrepaired cracked plate is validated comparing the obtained results with the analytical solution given by Newman and Raju, ${ }^{22}$ in which the SIF for an embedded semi-elliptical crack in a finite plate subjected to tension is defined by the following expression

$$
K_{I}=\sigma\left(\frac{\pi c}{Q}\right)^{1 / 2} F_{S}
$$

where the parameters of the equation are defined as

$$
\begin{gathered}
Q=1+1.464\left(\frac{c}{a}\right)^{1.65} \\
F_{S}=\left[M_{1}+M_{2}\left(\frac{c}{t}\right)^{2}+M_{3}\left(\frac{c}{t}\right)^{4}\right] g f_{\phi} f_{W}
\end{gathered}
$$

and

$$
\begin{gathered}
M_{1}=1.13-0.09 c / a \\
M_{2}=-0.54+\frac{0.89}{0.2+c / a} \\
M_{3}=0.5-\frac{1}{0.65+c / a}+14\left(1-\frac{c}{a}\right)^{24} \\
g=1+\left[0.1+0.35\left(\frac{c}{t}\right)^{2}\right](1-\sin \phi)^{2}
\end{gathered}
$$

and $f_{\phi}$ is given by

$$
f_{\phi}=\left[\left(\frac{c}{a}\right)^{2} \cos ^{2} \phi+\sin ^{2} \phi\right]^{1 / 4}
$$

The finite-width correction, $f_{W}$, has the form

$$
f_{W}=\left[\sec \left(\frac{\pi a}{2 b} \sqrt{\frac{c}{t}}\right)\right]^{1 / 2}
$$

All geometrical parameters and the coordinate system used to define the parametric angle, $\phi$, are defined in Figure 3. Note that equation (1) is valid for $0 \leq c / a \leq 2, a / b<0.5$, and $0 \leq \phi \leq \pi$.

The results of the SIF as a function of the parametric angle are presented in Figure 4. The length and depth of the crack are 12 and $1.5 \mathrm{~mm}$, respectively. The rest of parameters are commented in previous sections.
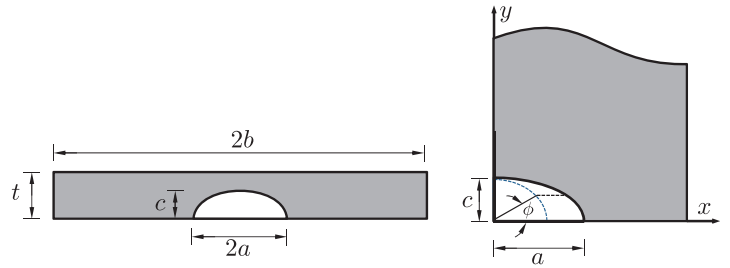

Figure 3. Representation of the geometrical parameters and coordinate system.

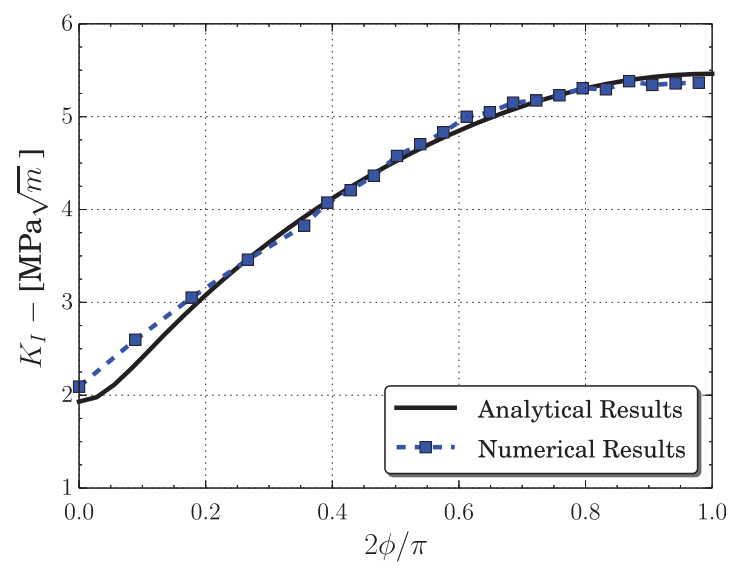

Figure 4. Comparison between analytical and numerical results for the obtained SIF.

Numerical SIF is very close to analytical SIF, and the difference between them is around $1 \%$ when the maximum value is reached. Thus, this confirms the adequacy of the mesh implemented, and similar procedure is considered for further analysis.

\section{Effect of the crack depth on the SIF variation}

Figure 5 presents the variation of the maximum normalised SIF for both single- and double-sided patches as a function of the crack depth $(c)$, normalised with the plate thickness $(t)$. The length of the crack $(a)$ is $12 \mathrm{~mm}$ and the radius of the patch is $50 \mathrm{~mm}$. The thickness of the composite laminate is $2 \mathrm{~mm}$ for the single-sided patch, and $1 \mathrm{~mm}$ for the double. The maximum normalised SIF is defined by

$$
\bar{K}_{I}=\frac{K_{I}}{K_{I}^{n p}}
$$

where $K_{I}$ and $K_{I}^{n p}$ respresent SIF for repaired crack and non-repaired crack, respectively.

According to the results presented in Figure 5, the performance of the double patch technique is independent of the crack depth, while the effectiveness of the single-patch decreases when the crack depth increases. 


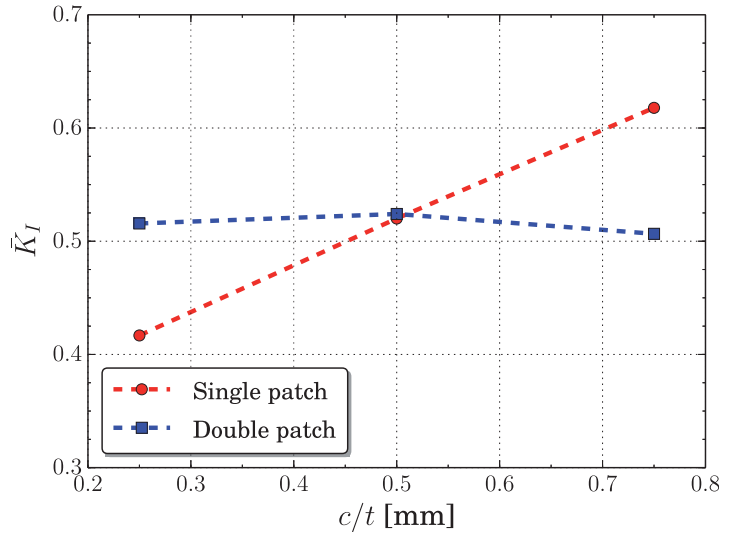

Figure 5. Maximum normalized SIF for single- and double-sided patch vs. normalised crack thickness.

Results show that the use of single-patched repairs is more effective when the crack depth is less than half the plate thickness, while the double-sided patch performs better when the normalised crack depth is more than 0.5 .

Therefore, the selection of single- or double-sided patches will depend on whether the crack depth is less or greater than half the plate thickness. Note that according to the results presented by Albedah et al., ${ }^{18}$ the use of a double-symmetric circular patch considerably increases the repair performance in thin-cracked plates having straight-crack front.

\section{Effect of the patch thickness on the SIF variation}

The structure studied in the previous section is now considered for different patch thickness $\left(e_{r}\right)$. Figure 6 shows the variation of the maximum normalised SIF as a function of the patch thickness for both single- and double-sided patches, and for different crack depths $(c=3,6$, and $9 \mathrm{~mm})$. In general, the performance of the repair increases with the thickness of the patch, presenting an asymptotic tendency to a limit of efficiency.

If the ratio of crack depth-to-plate thickness is small (Figure 6a), the thickness of the patch repair seems to be essential to improve the repair performance. For thinner repair patches $(<5 \mathrm{~mm})$, it is more useful to use a single-sided patch on the side in which the crack is visible than applying a double-sided, with half the thickness of that for the single-sided. However, single-sided patches prove to have limitations: normalised SIF increases for thicker patches. This could be due to the asymmetry of the repair on which the load is applied: thicker single-sided patches become more asymmetrical with respect to the face without patch. This asymmetry creates a bending field between patch and plate, leading to higher stresses in
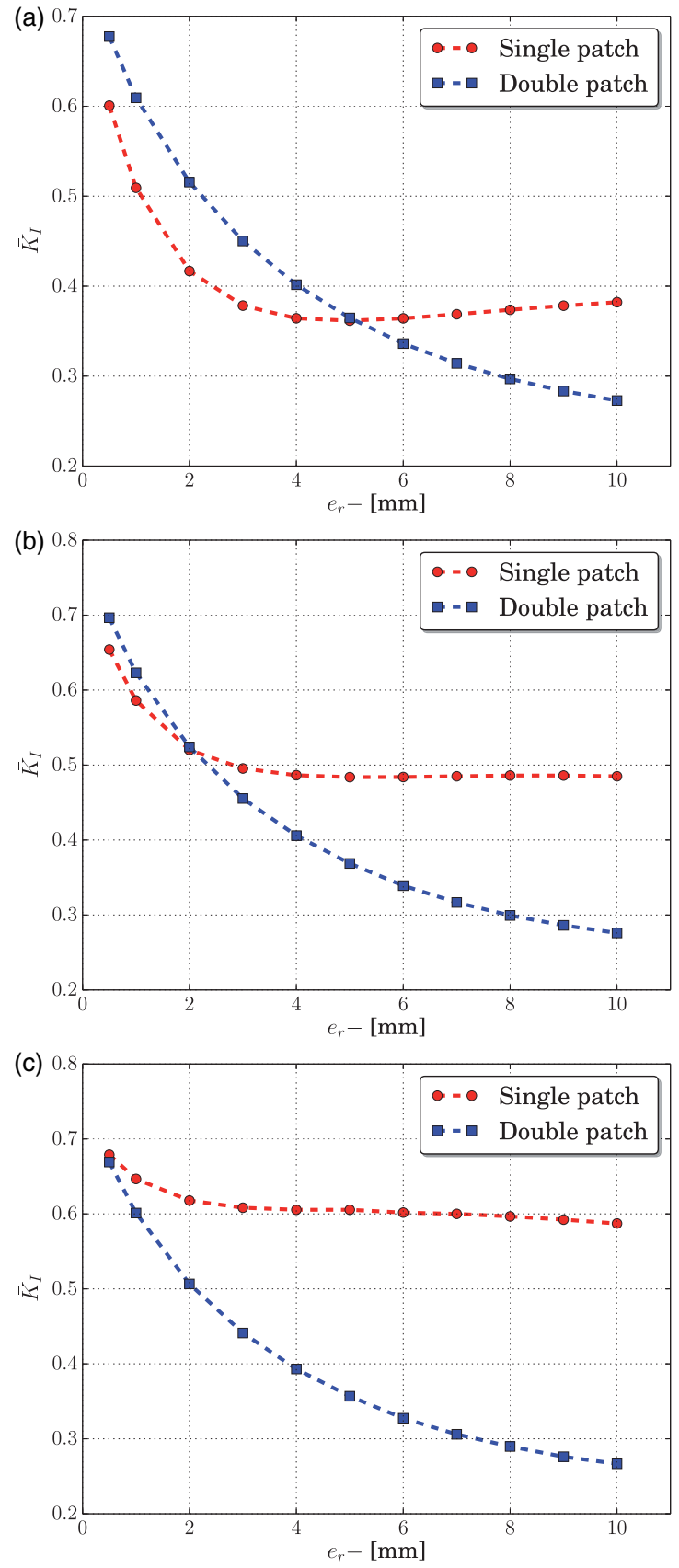

Figure 6. Maximum SIF for single- and double-sided patch vs. patch thickness: (a) $c / t=0.25$, (b) $c / t=0.5$, (c) $c / t=0.75$.

the vicinity of the crack. ${ }^{16}$ Similar results are obtained for surface cracks with depths equal to half the repaired plate thickness (Figure 6b). For surface cracks with depths greater than half the repaired plate thickness, the use of double-sided patches is recommended in all cases (Figure 6c) as the elliptic crack affects the stress distribution in the front and back sides.

Figure 7 presents the difference of the normalised SIF between single- and double-sided patch $\left(\Delta \bar{K}_{I}\right)$ as 


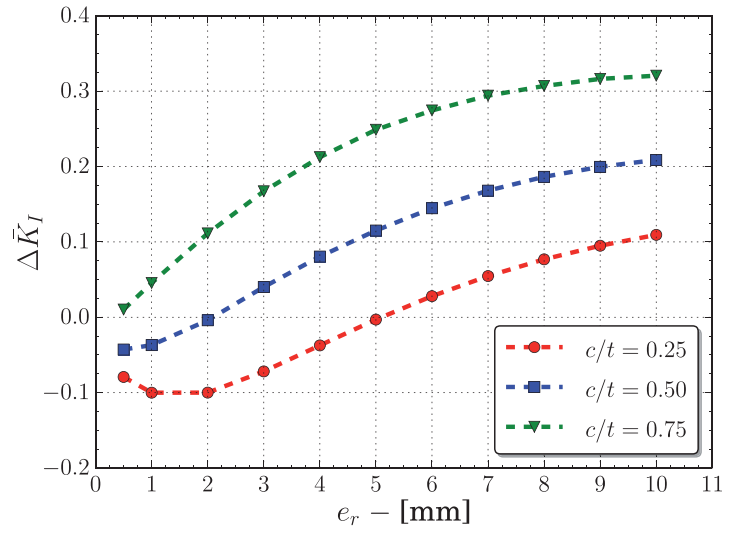

Figure 7. Difference of the maximum SIF between single- and double-sided patch vs. patch thickness.

a function of the patch thickness. This non-dimensional SIF is defined as

$$
\bar{K}_{I}=\frac{K_{I}^{s p}-K_{I}^{d p}}{K_{I}^{n p}}
$$

where $K_{I}^{s p}$ and $K_{I}^{d p}$ are the maximum SIF of the plate repaired with a single and double patch, respectively.

Note that negative values of $\Delta \bar{K}_{I}$ indicate that the single patch presents better performance than the double patch. The performance of the double-sided patch increases with respect to the single patch with increasing patch thickness or crack depth. Therefore, it is recommended to use the single-sided patch technique only when the crack depth is less than half the repaired plate thickness, and when the asymmetry of the patch is not high enough to decrease its performance.

\section{Effect of the patch material on the SIF variation}

Composite patch materials used in this study are boron/epoxy and T600/R368-1 carbon/epoxy. The mechanical properties of carbon/epoxy composite patches are presented in Table 2.

The effect of the variation of the composite patch material on the repair efficiency for single-sided and double-sided repair patches is presented in Figures 8 and 9, respectively. The results are presented as the difference of the normalised SIF between carbon/epoxy and boron/epoxy patch $\left(\Delta \bar{K}_{I}^{C B}\right)$ as a function of the patch thickness. This non-dimensional SIF is defined as

$$
\bar{K}_{I}^{C B}=\frac{K_{I}^{C}-K_{I}^{B}}{K_{I}^{n p}}
$$

where $K_{I}^{C}$ and $K_{I}^{B}$ are the maximum SIF of the plate repaired with a carbon/epoxy and boron/epoxy patch, respectively.
Table 2. Mechanical properties of composite T600/R368-I. ${ }^{27}$

\begin{tabular}{ll}
\hline$E_{11}(G P a)$ & 103 \\
$E_{22}=E_{33}(G P a)$ & 7 \\
$G_{12}=G_{13}(G P a)$ & 3.15 \\
$G_{23}(G P a)$ & 2.75 \\
$v_{12}$ & 0.34 \\
$v_{13}$ & 0.023 \\
$v_{23}$ & 0.30 \\
\hline
\end{tabular}

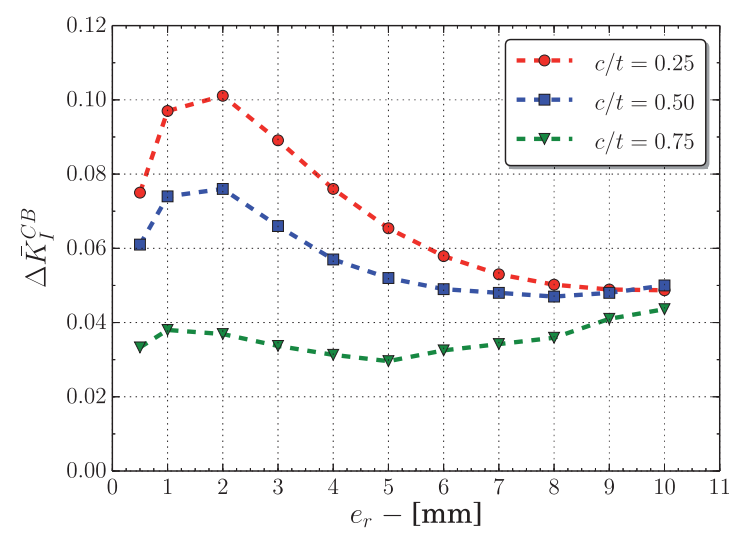

Figure 8. Difference of the maximum SIF between carbon/ epoxy and boron/epoxy single-sided patch vs. patch thickness.

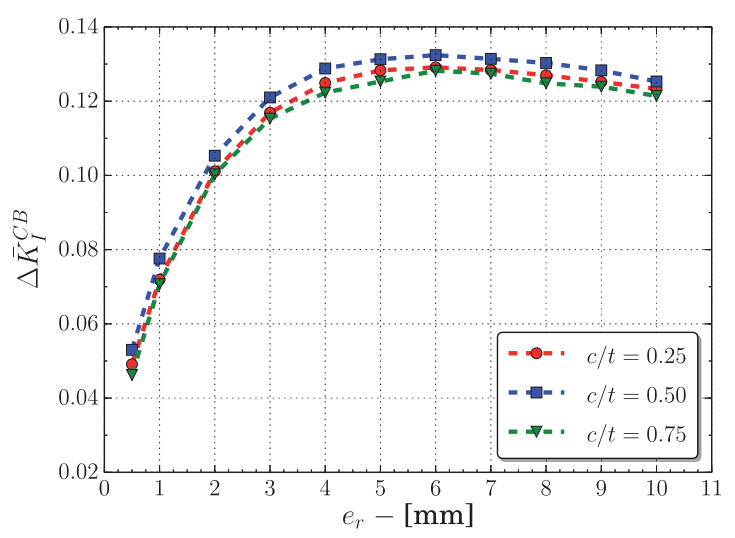

Figure 9. Difference of the maximum SIF between carbon/ epoxy and boron/epoxy double-sided patch vs. patch thickness.

Results show that the repair performance is strongly dependent on the composite patch material. Boron/ epoxy patches prove to be more efficent for all cases studied: $0.25,0.5$, and 0.75 crack depth-to-plate thickness ratios. If properties shown in Tables 1 and 2 are compared, it can be seen that boron/epoxy $\mathrm{E}_{11}$ is higher than carbon/epoxy $E_{11}$, thus this improvement in efficiency is quite evident. 
In double-sided repairs the non-dimensional SIF increases with increasing patch thickness for the three $\mathrm{c} / \mathrm{t}$ ratios studied. Results show similarities in value and trend. In single-sided repairs the non-dimensional SIF decreases with increasing patch thickness. Differences are more noticeable in single-sided repair patches.

\section{Conclusions}

In the present study, the influence of single- and double-sided adhesively-bonded composite patch repairs on the mechanical perfomance of thick aluminium plates with semi-elliptical surface cracks is analysed by simulation virtual tests using Abaqus/Standard finite-element code. The repaired cracked plate is subjected to uniaxial tensile load, and maximum value of the SIF in mode I is evaluated. The following parameters are studied: patch configuration (single- or double-sided), patch thickness, crack size, and patch material.

Results show that the choice between using single- or double- sided patches depends on the crack depth, and for surface cracks with depth less than half of the repaired plate thickness, single-sided patch configuration performace is better as compared to doublesided patches, as the crack affects more the side of plate where it is visible. As the ratio of crack depthto-plate thickness increases, it is recommended to use double-sided patch configuration to repair surface cracks. In all cases, the performance of the patches increases with the thickness of the composite patch. However, due to the increasing asymmetry of singlesided patches with increasing thickness, for surface cracks with depths greater than half the repaired plate thickness, the use of double-sided patches is recommended.

The composite material used for the patch repairs present an important influence on the SIF results, being more noticeable in double-sided patch repairs.

The analysis presented in this work should be useful in predicting the SIF associated to surface cracks with semi-elliptical crack fronts, and in choosing between the use of the single- and double-sided patch configuration depending on the crack dimensions and depth.

\section{Declaration of Conflicting Interests}

The author(s) declared no potential conflicts of interest with respect to the research, authorship, and/or publication of this article.

\section{Funding}

The author(s) disclosed receipt of the following financial support for the research, authorship, and/or publication of this article: The Ministry of Economy and Competitiveness of Spain (project DPI2013-42240-R).

\section{References}

1. Baker A and Jones R. Bonded repair of aircraft structures. Dordrecht: Martinus Nijhoff Publishers, 1988.

2. Baker A and Chester RJ. Recent advances in bonded composite repair technology for metallic aircraft components. In: Proceedings of the international conference on advanced composite materials, 1993, pp.45-49.

3. Duong $\mathrm{CN}$ and Wang $\mathrm{CH}$. Composite repair: theory and design. Oxford: Elsevier Science Ltd, 2007.

4. Pantelakis S and Tserpes KI. Adhesive bonding of composite aircraft structures: challenges and recent developments. Sci China-Phys Mech Astron 2014; 57: 2011.

5. Catalanotti G, Camanho PP, Xavier J, et al. Measurement of resistance curves in the longitudinal failure of composites using digital image correlation. Compos Sci Technol 2010; 70: 1986-1993.

6. Bachir Bouiadjra B, Fari Bouanani M, Albedah A, et al. Comparison between rectangular and trapezoidal bonded composite repairs in aircraft structures: a numerical analysis. Mater Des 2011; 32: 3161-3166.

7. $\mathrm{Hu}$ FZ and Soutis C. Strength prediction of patchrepaired CFRP laminates loaded in compression. Compos Sci Technol 2000; 60: 1103-1114.

8. Bachir Bouiadjra B, Oudad W, Albedah A, et al. Effects of the adhesive disband on the performances of bonded composite repairs in aircraft structures. Mat Des 2012; 37: 89-95.

9. Ramji M, Srilakshmi R and Bhanu Prakash M. Towards optimization of patch shape on the performance of bonded composite repair using FEM. Compos Part B 2013; 45: 710-720.

10. Benyahia F, Aminallah L, Albedah A, et al. Experimental and numerical analysis of bonded composite patch repair in aluminum alloy 7075 T6 F. Mater Des 2015; 73: 67-73.

11. Xiaocong H. A review of finite element analysis of adhesively bonded joints. Int $J$ Adhes Adhes 2011; 31: 248-264.

12. Fari Bouanani M, Benyahia F, Albedah A, et al. Analysis of the adhesive failure in bonded composite repair of aircraft structures using modified damage zone theory. Mater Des 2013; 50: 433-439.

13. Bachir Bouiadjra B, Belhouari $M$ and Serier $B$. Computation of the stress intensity factor for repaired cracks with bonded composite patch in mode I and mixed mode. Compos Struct 2002; 56: 401-406.

14. Benyahia F, Albedaha A and Bachir Bouiadjra BA. Elliptical and circular bonded composite repair under mechanical and thermal loading in aircraft structures. Mat Res 2014; 17: 1219-1225.

15. Ouinas D, Bouiadjra BB, Serier B, et al. Comparison of the effectiveness of boron/epoxy and graphite/ epoxy patches for repaired cracks emanating from a semicircular notch edge. Compos Struct 2007; 80: 514-522.

16. Ramji M and Srilakshmi R. Design of composite patch reinforcement applied to mixed-mode cracked panel using finite element analysis. $J$ Reinf Plast Compos 2012; 31: 585-595.

17. Ouinas D, Sahnoune M, Benderdouche N, et al. Stress intensity factor analysis for notched cracked structure 
repaired by composite patching. Mater Des 2009; 30: 2302-2308.

18. Albedah A, Bachir Bouiadjra B, Mhamdia R, et al. Comparison between double and single sided bonded composite repair with circular shape. Mater Des 2011; 32: 996-1000.

19. Bachir Bouiadjra B, Ouinas D, Serier B, et al. Disbond effects on bonded boron/epoxy composite repair to aluminium plates. Comput Mater Sci 2008; 42: 220-227.

20. Kwon W and Hall BL. Analyses of cracks in thick stiffened plates repaired with single-sided composite patch. Compos Struct 2015; 119: 727-737.

21. Tsai GC and Shen SB. Fatigue analysis of cracked thick aluminum plate bonded with composite patches. Compos Struct 2004; 64: 79-90.

22. Newman JC Jr and Raju IS. Stress-intensity factor equations for cracks in three-dimensional finite bodies. In: Fracture mechanics: fourteenth symposium. Volume I: theory and analysis, ASTM STP 791, American Society for Testing and Materials, Philadelphia, 1983, pp.238-265.

23. Srilakshmi R, Ramji $M$ and Chinthapenta V. Fatigue crack growth study of CFRP patch repaired Al 2014-T6 panel having an inclined center crack using FEA and DIC. Eng Fract Mech 2015; 134: 182-201.

24. Gran RJ, Orazio FD, Paris PC, et al. Investigation and analysis development of early life aircraft structural failures, AFFDL-TR-70-149, US Air Force Flight Dynamics Laboratory, 1971.

25. Jones R and Chiu WK. Composites repairs to cracks in thick metallic components. Compos Struct 1999; 44: 17-29.

26. Abaqus/Standard version 6.13. User's manual. Hibbit, Karlsson: 2013.

27. Kashfuddoja M and Ramji M. Design of optimum patch shape and size for bonded repair on damaged Carbon fibre reinforced polymer panels. Mater Des 2014; 54: 174-183. 